\title{
Molecular clumps and star formation associated with the infrared dust bubble N131
}

\author{
C.-P. Zhang ${ }^{1,2,3}$, J.-J. Wang ${ }^{1,2}$, and J.-L. Xu ${ }^{1,2}$ \\ 1 National Astronomical Observatories, Chinese Academy of Sciences, 100012 Beijing, PR China \\ e-mail: zcp0507@gmail.com \\ 2 NAOC - TU Joint Center for Astrophysics, 850000 Lhasa, PR China \\ 3 University of the Chinese Academy of Sciences, 100080 Beijing, PR China
}

Received 13 August 2012 / Accepted 19 December 2012

\section{ABSTRACT}

\begin{abstract}
Aims. The aim is to explore the interstellar medium around the dust bubble N131 and search for signatures of star formation. Methods. We have performed a multiwavelength study around the N131 with data taken from large-scale surveys of infrared observation with online archive. We present new observations of three $\mathrm{CO} J=1-0$ isotope variants from Purple Mountain Observatory $13.7 \mathrm{~m}$ telescope. We analyzed the distribution of the molecular gas and dust in the environment of the N131. We used color-color diagrams to search for young stellar objects and to identify ionizing star candidates.

Results. The kinematic distance of $\sim 8.6 \mathrm{kpc}$ has been adopted as the distance of the bubble N131 from the Sun in this work. We find a ring of clouds in CO emission coincident with the shell of N131 seen in the Spitzer telescope images, and two giant elongated molecular clouds of $\mathrm{CO}$ emission appearing on opposite sides of the ringlike shell of N131. There is a cavity within the bubble at $1.4 \mathrm{GHz}$ and $24 \mu \mathrm{m}$. Seven IRAS point sources are distributed along the ringlike shell of the bubble N131. Fifteen ionizing stars and 63 YSO candidates have been found. The clustered class I and II YSOs are distributed along the elongated clouds in the line of sight.
\end{abstract}

Key words. infrared: ISM - stars: formation - ISM: bubbles - HII regions

\section{Introduction}

Churchwell et al. $(2006,2007)$ have detected and cataloged about 600 midinfrared dust (MIR) bubbles between longitudes $-60^{\circ}$ and $+60^{\circ}$. The IR dust bubbles may be produced by ionizing $\mathrm{O}$ - and/or B-type stars, which are located inside the bubble. The ultraviolet (UV) radiation from ionizing stars may heat dust and ionize the gas to form an expanding bubble shell (Watson et al. 2008). Simpson et al. (2012) present a new catalog of 5106 infrared bubbles created through visual classification via the online citizen science website "The Milky Way Project" 1 , which provides a crowd-sourced map of bubbles and arcs in the Milky Way, and will enable better statistical analysis of Galactic star-forming sites. Beaumont \& Williams (2010) report CO $J=3-2$ maps of 43 Spitzer identified bubbles, which suggests that expanding shock fronts are poorly bound by molecular gas and that cloud compression by these shocks may be limited. Watson et al. (2008) present an analysis of wind-blown, parsec-sized, midinfrared bubbles, and associated star formation, and suggest that more than a quarter of the bubbles may have triggered the formation of massive objects.

A few individual bubbles have been studied well, such as N22 (Ji et al. 2012), N49 (Watson et al. 2008; Deharveng et al. 2010; Zavagno et al. 2010), N65 (Petriella et al. 2010), N68 (Zhang \& Wang 2012b), and S51 (Zhang \& Wang 2012a). There are many models and observations to explain the dusty windblown bubbles, such as bubble N49 of Everett \& Churchwell (2010). Recently, we have reported an expanding ringlike shell of the bubble S51, which shows a rare front side located within

\footnotetext{
1 http://www.milkywayproject.org
}

the shell in the line of sight, by employing ${ }^{13} \mathrm{CO}$ and $\mathrm{C}^{18} \mathrm{O} J=$ 1-0 emission lines of the Mopra Telescope (Zhang \& Wang 2012a). We also investigated the star formation around the bubble N68, which suggests that the massive star formation at the ringlike shell is very active (Zhang \& Wang 2012b).

Toward the bubble N131, in this work, we carried out new observations of the $J=1-0$ transitions of ${ }^{12} \mathrm{CO},{ }^{13} \mathrm{CO}$, and $\mathrm{C}^{18} \mathrm{O}$ using the telescope of the Purple Mountain Observatory (PMO) at Qinghai province in China. we mainly report several molecular clumps associated well with the IR dust bubble N131. We also aim to explore its surrounding ISM and search for star formation spots. We describe the data in Sect. 2; the results and discussion are presented in Sect. 3; Sect. 4 summarizes the results.

\section{Observation and data processing}

\subsection{The online archive}

The data of the online archive used in this work include GLIMPSE (Benjamin et al. 2003; Churchwell et al. 2009), MIPSGAL (Carey et al. 2009), the Two Micron All Sky Survey $(2 \mathrm{MASS})^{2}$ (Skrutskie et al. 2006), IRAS (Neugebauer et al. 1984), and NVSS (Condon et al. 1998). GLIMPSE is an MIR survey of the inner Galaxy performed with the Spitzer Space Telescope. We used the mosaicked images from GLIMPSE and the GLIMPSE Point-Source Catalog (GPSC) in the Spitzer$\operatorname{IRAC}(3.6,4.5,5.8$, and $8.0 \mu \mathrm{m})$. IRAC has an angular resolution

\footnotetext{
2 2MASS is a joint project of the University of Massachusetts and the Infrared Processing and Analysis Center/California Institute of Technology, funded by the National Aeronautics and Space Administration and the National Science Foundation.
} 
between 1.5" and 1.9" (Fazio et al. 2004; Werner et al. 2004). MIPSGAL is a survey of the same region as GLIMPSE, using the MIPS instrument ( 24 and $70 \mu \mathrm{m})$ on Spitzer. The MIPSGAL resolution is 6" at $24 \mu \mathrm{m}$. The IRAS Point Source Catalog consists of 245889 sources found and verified by the IRAS (InfraRed Astronomy Satellite) at 12, 25, 60, and $100 \mu \mathrm{m}$. The NRAO VLA Sky Survey (NVSS) is a $1.4 \mathrm{GHz}$ continuum survey covering the entire sky north of $-40^{\circ}$ declination (Condon et al. 1998); the NVSS survey has a noise of about $0.45 \mathrm{mJy}^{\text {beam }}{ }^{-1}$.

\subsection{The CO data of Purple Mountain Observatory}

Our CO observations were made during May 2012 using the 13.7-m millimeter telescope of Qinghai Station at the Purple Mountain Observatory at Delingha ${ }^{3}$. We used the nine-pixel array receiver separated by $\sim 180^{\prime \prime}$. The receiver was operated in the sideband separation of single sideband mode, which allows for simultaneous observations of three $\mathrm{CO} J=1-0$ isotope variants, with ${ }^{12} \mathrm{CO}$ in the upper sideband (USB) and ${ }^{13} \mathrm{CO}$ and $\mathrm{C}^{18} \mathrm{O}$ in the lower sideband (LSB). The half-power beam width (HPBW) is $52^{\prime \prime} \pm 3^{\prime \prime}$, and the main beam efficiency is $\sim 50 \%$ at $\sim 110 \mathrm{GHz}$. The pointing and tracking accuracies are better than $5^{\prime \prime}$. The typical system temperature during our runs was around $110 \mathrm{~K}$ and varies by about $10 \%$ for each beam. A fast Fourier transform (FFT) spectrometer was used as the back end with a total bandwidth of $1 \mathrm{GHz}$ and 16384 channels. The velocity resolution is about $0.16 \mathrm{~km} \mathrm{~s}^{-1}$ at $\sim 110 \mathrm{GHz}$.

On-the-fly (OTF) observing mode was applied for mapping observations. The antenna continuously scanned a region of $20^{\prime} \times 20^{\prime}$ centered on $\mathrm{RA}(\mathrm{J} 2000)=19^{\mathrm{h}} 52^{\mathrm{m}} 09^{\mathrm{s}} \cdot 61, \operatorname{Dec}(\mathrm{J} 2000)=$ $26^{\circ} 22^{\prime} 13^{\prime \prime} 4$ with a scan speed of $20^{\prime \prime} \mathrm{s}^{-1}$. The OFF position was chosen at $\mathrm{RA}(\mathrm{J} 2000)=19^{\mathrm{h}} 47^{\mathrm{m}} 24 \mathrm{~s} .00, \operatorname{Dec}(\mathrm{J} 2000)=$ $28^{\circ} 29^{\prime} 24^{\prime \prime} 0$, where there is extremely weak CO emission based on the CO survey of the Milky Way (Dame et al. 1987, 2001). The rms noise level was $0.2 \mathrm{~K}$ in main beam antenna temperature $T_{\mathrm{A}}^{*}$ for ${ }^{12} \mathrm{CO}(1-0)$, and $0.1 \mathrm{~K}$ for ${ }^{13} \mathrm{CO}(1-0)$ and $\mathrm{C}^{18} \mathrm{O}(1-0)$. The OTF data were then converted to 3 -D cube data with a grid spacing of 30". The IRAM software package GILDAS ${ }^{4}$ and the software package MIRIAD ${ }^{5}$ were used for the data reduction.

\section{Analysis and the results}

\subsection{The dimension and distance of the bubble N131}

We selected the IR dust bubble N131 from the catalog of Churchwell et al. (2006). They suggest that the N131 is a complete (closed ring) IR dust bubble centered on $l^{*}=63.084, b^{*}=$ -0.395 , with an inner short radius $r_{\text {in }}^{*}=5.46^{\prime}$, an inner long radius $R_{\text {in }}^{*}=6.18^{\prime}$, and an eccentricity of the ellipse $e_{\mathrm{N} 131}^{*}=0.47$. By comparing the IR emission with the integrated intensity of $\mathrm{CO}$ emission, the dimensions about the ring of cloud are respectively $r_{\text {in }}=5.20^{\prime}, R_{\text {in }}=6.00^{\prime}$, and $e_{\mathrm{N} 131}=0.50$ centered on $l=$ $63.095, b=-0.404$, or $\mathrm{RA}(\mathrm{J} 2000)=19^{\mathrm{h}} 52^{\mathrm{m}} 21^{\mathrm{s}} .5, \operatorname{Dec}(\mathrm{J} 2000)=$ $+26^{\circ} 21^{\prime} 24^{\prime \prime} .0$. Here, $r_{\text {in }}$ and $R_{\text {in }}$ are the semiminor and semimajor axes of the inner ellipse, respectively. By analyzing $\mathrm{H}_{2} \mathrm{CO}$ absorption $\left(V_{\mathrm{H}_{2} \mathrm{CO}}=22.6 \pm 0.1 \mathrm{~km} \mathrm{~s}^{-1}\right)$ against the UC HII region continuum emission $\left(V_{\mathrm{H} 110 \alpha}=-9.3 \pm 2.3 \mathrm{~km} \mathrm{~s}^{-1}\right)$, Watson et al. (2003) resolved the distance ambiguity toward G63.05-0.34, which lies on the "far" kinematic distance $8.6_{-1.0}^{+0.9} \mathrm{kpc}$. In fact,

\footnotetext{
3 http://www.dlh.pmo.cas.cn/

4 http://iram.fr/IRAMFR/GILDAS/

5 http://www.cfa.harvard.edu/sma/miriad/
}

there is no distance ambiguity toward N131, because the derived "near" kinematic distance is negative. The G63.05-0.34 is located at the position of the IRAS $19499+2613$, which is correlated with $\mathrm{CO}$ molecular clump $\mathrm{A}\left(V_{\mathrm{CO}}=\sim-10.5 \mathrm{~km} \mathrm{~s}^{-1}\right)$ in this work. Watson et al. (2010), however, adopted the velocity of $\mathrm{H}_{2} \mathrm{CO}\left(\sim 22.6 \mathrm{~km} \mathrm{~s}^{-1}\right)$ in Watson et al. (2003) to obtain a kinematic distance of $\sim 2.4 \mathrm{kpc}$ toward the bubble N131. Actually, there is another $\mathrm{CO}$ velocity component at $\sim 25.0 \mathrm{~km} \mathrm{~s}^{-1}$, which is consistent with $\mathrm{H}_{2} \mathrm{CO}$ velocity at $\sim 22.6 \mathrm{~km} \mathrm{~s}^{-1}$, but not correlated with the ringlike shell of this bubble. This velocity component of $\sim 22.6 \mathrm{~km} \mathrm{~s}^{-1}$ may belong to the foreground of the bubble N131. Therefore, we adopt the kinematic distance $D_{\mathrm{N} 131}=$ $8.6 \mathrm{kpc}$ as the distance of the bubble N131. The dimensions of the inner short radius and the inner long radius are $D_{r_{\text {in }}}=13.0 \mathrm{pc}$ and $D_{R_{\mathrm{in}}}=15.0 \mathrm{pc}$, respectively.

\subsection{Parameters of the spectra and molecular clumps}

Figure 1 shows several spectra for ${ }^{12} \mathrm{CO},{ }^{13} \mathrm{CO}$, and $\mathrm{C}^{18} \mathrm{O}$ at the peak locations of nine molecular clumps from $\mathrm{A}$ to I (indicated in Fig. 2) defined on the basis of ${ }^{13} \mathrm{CO}$ contours. For the nine molecular clumps, we detected strong ${ }^{12} \mathrm{CO}$ and ${ }^{13} \mathrm{CO}$ emission lines. At any position toward the N131, however, we did not detect any $\mathrm{C}^{18} \mathrm{O}$ emission signal of more than $3 \sigma$. We reported spectral information of the nine molecular clumps in Table 1, and some derived parameters of the molecular clumps in Table 2. In Table 1, Col. (1) lists the molecular clump name; Cols. (2), (3) list the equatorial coordinates; Cols. (4)-(9) list the LSR velocity $V_{\mathrm{CO}}$, the full width at half maximum (FWHM) $\Delta V_{\mathrm{CO}}$, and the peak brightness temperature $T_{\mathrm{CO}}$ of each clump using Gaussian fitting for $\mathrm{CO}$ emission line. In Table 2, Cols. (1)-(4) show the name, the dimension, and the integration temperature of each molecular clump, where the area of each clump is indicated with a dashed ellipse and polygon in Fig. 2; Cols. (5)-(8) show the maximum and mean value of the ionizing temperature and the $\mathrm{H}_{2}$ column density; Cols. (9)-(10) show the mass and density of each molecular clump. Here, we assumed each molecular clump is under the local thermal equilibrium (LTE) assumption, and used the theory of the radiation transfer and molecular excitation (Winnewisser et al. 1979; Garden et al. 1991). The excitation temperature $T_{\text {ex }}$ and column density $N_{{ }_{13} \mathrm{CO}}$ can be calculated directly, assuming ${ }^{12} \mathrm{CO}$ emission to be optically thick and the beam-filling factor to be unity. The column densities of $\mathrm{H}_{2}$ were obtained by adopting typical abundance ratios $\left[\mathrm{H}_{2}\right] /\left[{ }^{12} \mathrm{CO}\right]=10^{4}$ and $\left[{ }^{12} \mathrm{CO}\right] /\left[{ }^{13} \mathrm{CO}\right]=60$ in the ISM.

\subsection{The ringlike shell}

In Fig. 2, the $8.0 \mu \mathrm{m}$ emission colorscale outlines clearly the distribution of molecular filament and clumpy structure toward the bubble N131. The $8.0 \mu \mathrm{m}$ emission originates mainly in the polycyclic aromatic hydrocarbons (PAHs). Inside the N131, the $8.0 \mu \mathrm{m}$ emission is much weaker than that at the ringlike shell. It is likely that the stellar wind from O- and/or early B-type stars have blown the PAHs onto the ringlike shell. In Fig. 3, the colorscale is the MIPSGAL $24 \mu \mathrm{m}$ emission. Generally, there should be strong $24 \mu \mathrm{m}$ emission and $1.4 \mathrm{GHz}$ continuum emission inside bubble, such as in the dust bubbles S51 (Zhang \& Wang 2012a) and N68 (Zhang \& Wang 2012b). However, having nearly the same distribution as $8.0 \mu \mathrm{m}$ emission, the $24 \mu \mathrm{m}$ emission is very weak inside the bubble N131.

Comparing ${ }^{12} \mathrm{CO}$ emission in Fig. 2 with ${ }^{13} \mathrm{CO}$ emission in Fig. 3, we can find that several molecular clumps were connected 

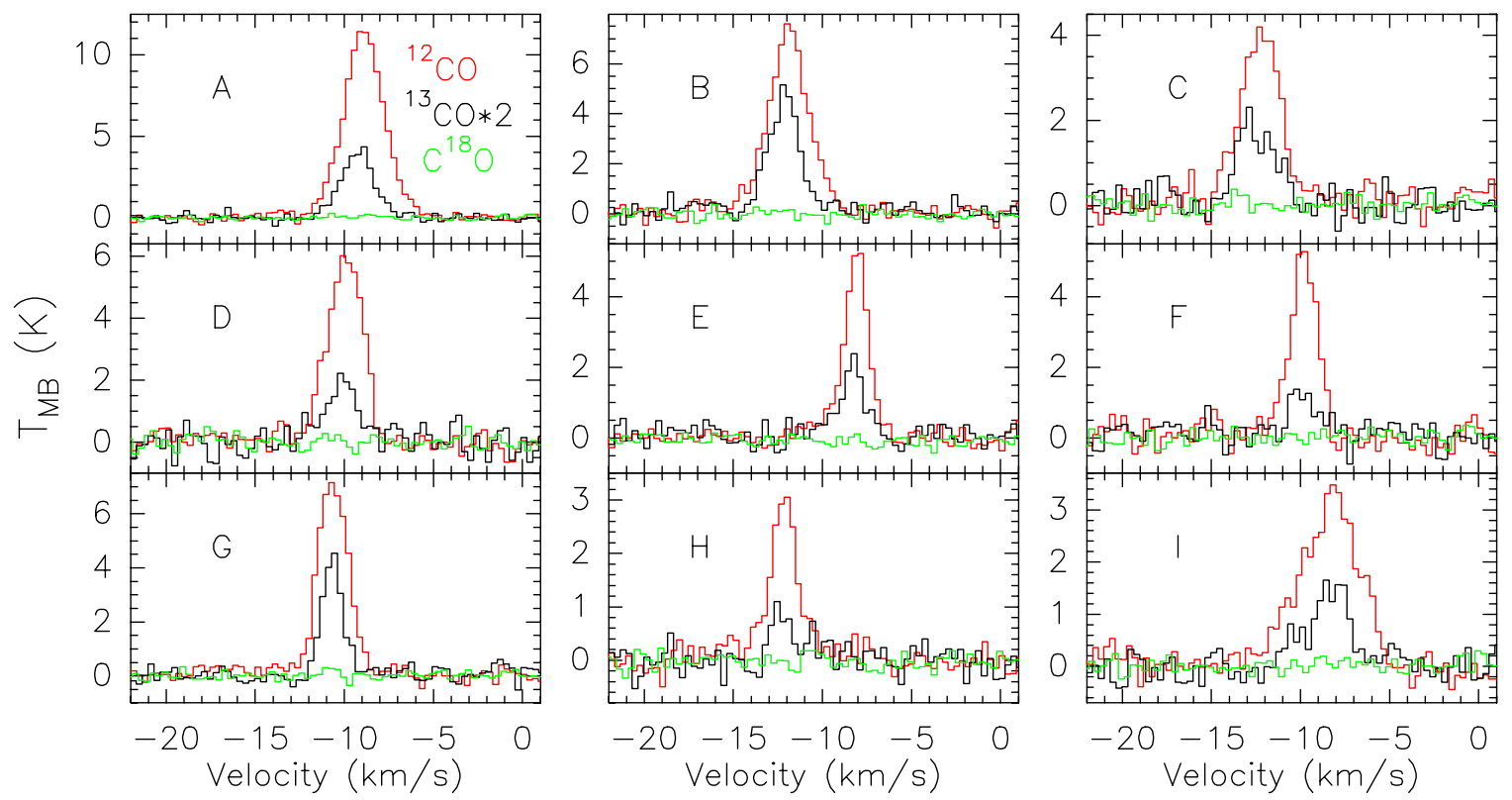

Fig. 1. ${ }^{12} \mathrm{CO}$ (red line), ${ }^{13} \mathrm{CO}$ (black line), and $\mathrm{C}^{18} \mathrm{O}$ (green line) spectra at the peaks of the molecular clumps from A to I. The brightness temperature of each ${ }^{13} \mathrm{CO}$ spectrum is multiplied by 2.

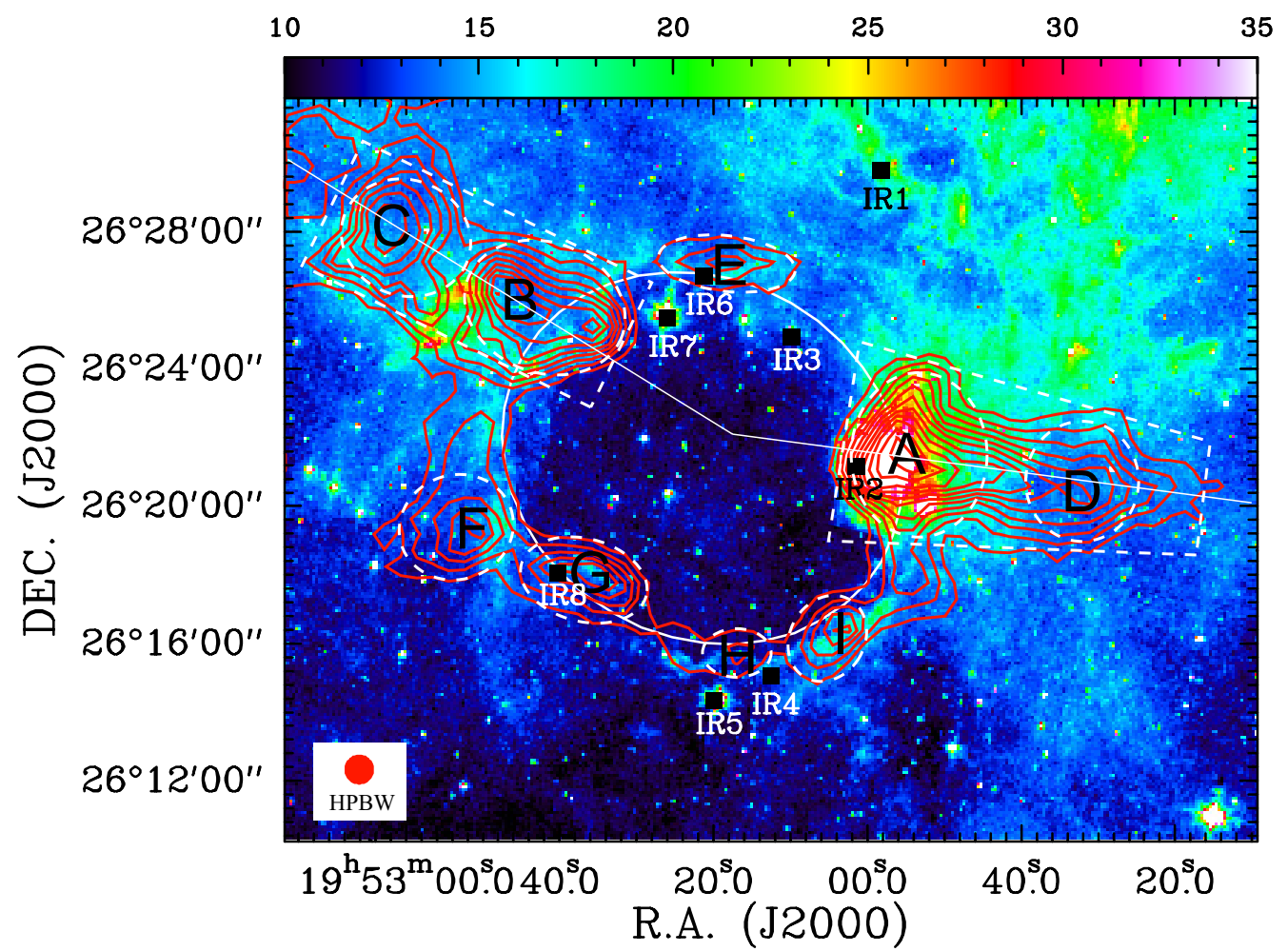

Fig. 2. Integrated intensity contours of the ${ }^{12} \mathrm{CO}$ emission clouds superimposed on the GLIMPSE $8.0 \mu \mathrm{m}$ colorscale. The contour levels range from 5.35 to 32.11 by $2.68 \mathrm{~K} \mathrm{~km} \mathrm{~s}^{-1}$. The integration range is from -14.5 to $-6.5 \mathrm{~km} \mathrm{~s}^{-1}$. The letters from A to I indicate the positions of nine molecular clumps, and the area of each clump is indicated with dashed ellipse and polygon. The black symbols " $\square$ " indicate the positions of IRAS point sources. The white ellipse indicates the position of bubble N131, and the straight lines indicate the position of position-velocity diagram in Fig. 5. The beam size of ${ }^{12} \mathrm{CO}$ emission is given in the filled red circle. The unit of the color bar is in $\mathrm{MJy} \mathrm{sr}^{-1}$.

together to form the ringlike shell of the N131. The inner edge of the ringlike shell has a much sharper gradient of ${ }^{12} \mathrm{CO}$ emission than the outer edge. Based on the distribution of optically thin ${ }^{13} \mathrm{CO}$ in Fig. 3, we also found seven clumps A, B, E, F, ..., and I along the ringlike shell. The parameters of the clumps in Table 2 show that the dense cores of star formation may be forming in clumps. Therefore, the ringlike shell is a possible birth place of star formation that was triggered by the bubble N131.

\subsection{Two elongated molecular clouds}

In Fig. 2, we also found there are two giant elongated molecular clouds (AD and BC) of CO emission appearing on opposite sides of the ringlike shell of N131. Morphologically, each cloud is in alignment, and the central axis of each cloud nearly goes through the center of the N131. The densest position of each cloud is located at the ringlike shell of the N131, and the two clouds AD 


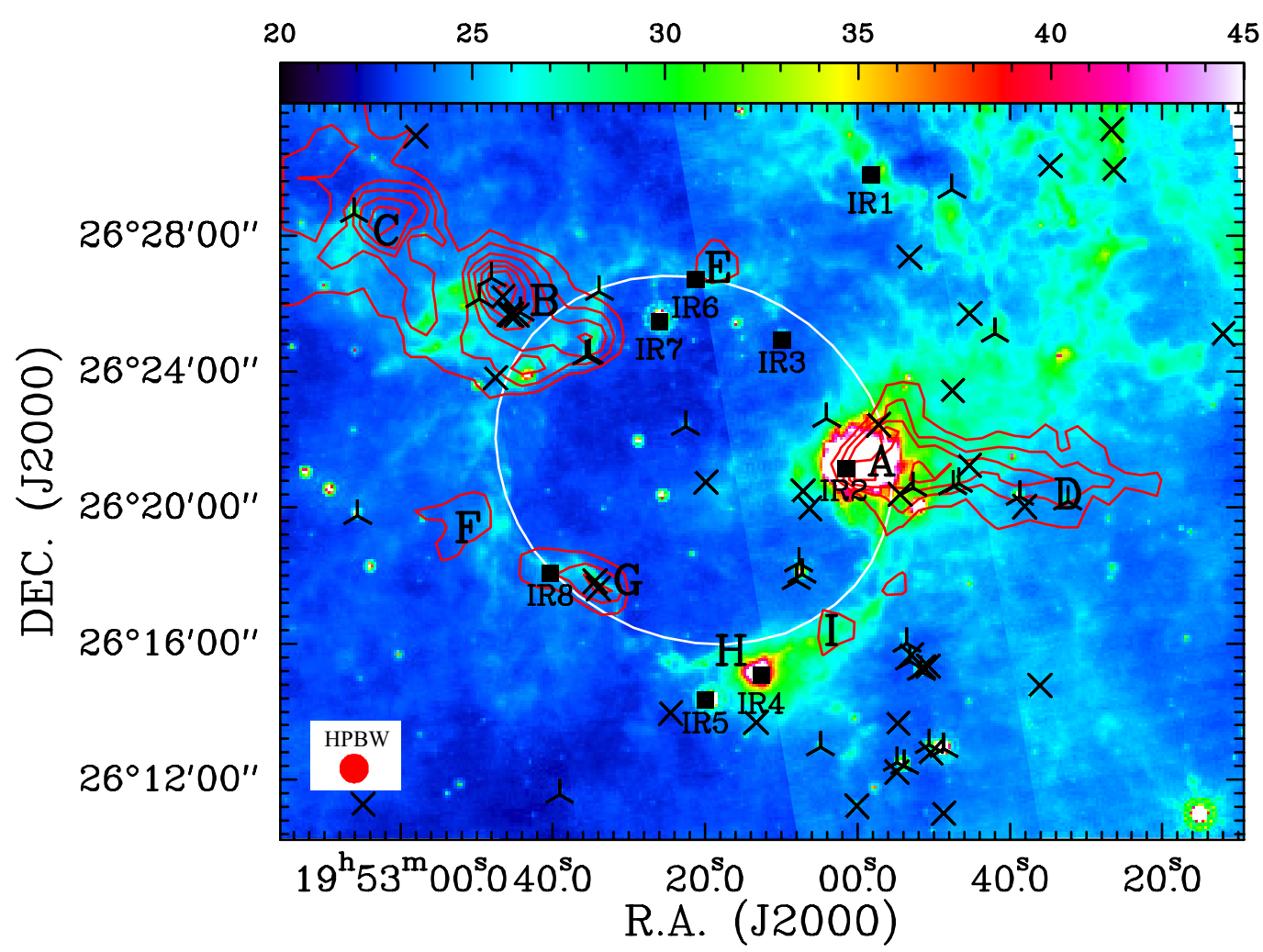

Fig. 3. Integrated intensity contours of the ${ }^{13} \mathrm{CO}$ emission clouds superimposed on the MIPSGAL $24 \mu \mathrm{m}$ colorscale. The contour levels range from 2.17 to 8.68 by $1.09 \mathrm{~K} \mathrm{~km} \mathrm{~s}^{-1}$. The integration range is from -14.5 to $-6.5 \mathrm{~km} \mathrm{~s}^{-1}$. The letters from A to I indicate the positions of nine molecular clumps, and the symbols "ᄉ”, “ $\times$ ", and "回" indicate the positions of class I, class II, and IRAS point sources, respectively. The white ellipse indicates the position of bubble N131. The beam size of ${ }^{12} \mathrm{CO}$ emission is given in the filled red circle. The unit of the color bar is in $\mathrm{MJy} \mathrm{sr}^{-1}$.

Table 1. Parameters of the nine spectra.

\begin{tabular}{|c|c|c|c|c|c|c|c|c|}
\hline Clump & $\begin{array}{c}\mathrm{RA}(\mathrm{J} 2000) \\
\mathrm{h} \quad \mathrm{s} \\
(2)\end{array}$ & $\begin{array}{c}\operatorname{Dec}(\mathrm{J} 2000) \\
,^{\prime}, " \\
(3)\end{array}$ & $\begin{array}{c}V_{12} \mathrm{CO}^{-1} \\
\left(\mathrm{~km} \mathrm{~s}^{-1}\right) \\
(4)\end{array}$ & $\begin{array}{c}V_{13} \mathrm{CO}^{-2} \\
\left(\mathrm{~km} \mathrm{~s}^{-2}\right) \\
(5)\end{array}$ & $\begin{array}{c}\Delta V_{12} \mathrm{CO} \\
\left(\mathrm{km} \mathrm{s}^{-1}\right) \\
(6)\end{array}$ & $\begin{array}{c}\Delta V_{13} \mathrm{CO} \\
\left(\mathrm{km} \mathrm{s}^{-2}\right) \\
(7)\end{array}$ & $\begin{array}{c}T_{12} \mathrm{CO} \\
(\mathrm{K}) \\
(8)\end{array}$ & $\begin{array}{c}T_{13} \mathrm{CO} \\
(\mathrm{K}) \\
(9)\end{array}$ \\
\hline A & 195155.681 & 262129.87 & $-8.91 \pm 0.01$ & $-9.25 \pm 0.03$ & $2.63 \pm 0.02$ & $2.14 \pm 0.06$ & $11.40 \pm 0.25$ & $2.04 \pm 0.13$ \\
\hline B & 195245.568 & 262554.58 & $-11.89 \pm 0.02$ & $-12.15 \pm 0.03$ & $2.65 \pm 0.05$ & $2.05 \pm 0.07$ & $7.19 \pm 0.31$ & $2.38 \pm 0.16$ \\
\hline $\mathrm{C}$ & 195301.891 & 262806.93 & $-12.24 \pm 0.04$ & $-12.53 \pm 0.08$ & $2.62 \pm 0.09$ & $2.53 \pm 0.17$ & $4.13 \pm 0.26$ & $0.95 \pm 0.16$ \\
\hline D & 195132.383 & 262022.06 & $-9.93 \pm 0.02$ & $-10.15 \pm 0.10$ & $2.30 \pm 0.05$ & $2.23 \pm 0.29$ & $6.07 \pm 0.34$ & $0.95 \pm 0.19$ \\
\hline E & 195218.309 & 262702.22 & $-8.09 \pm 0.03$ & $-8.29 \pm 0.05$ & $1.55 \pm 0.09$ & $1.63 \pm 0.17$ & $5.22 \pm 0.23$ & $1.02 \pm 0.16$ \\
\hline $\mathrm{F}$ & 195251.126 & 261923.40 & $-9.80 \pm 0.03$ & $-9.84 \pm 0.12$ & $1.77 \pm 0.06$ & $2.05 \pm 0.37$ & $5.25 \pm 0.36$ & $0.62 \pm 0.16$ \\
\hline G & 195235.316 & 261801.05 & $-10.64 \pm 0.01$ & $-10.68 \pm 0.02$ & $1.97 \pm 0.03$ & $1.33 \pm 0.05$ & $7.35 \pm 0.31$ & $2.24 \pm 0.12$ \\
\hline $\mathrm{H}$ & 195216.594 & 261545.75 & $-12.15 \pm 0.03$ & $-12.36 \pm 0.10$ & $1.82 \pm 0.08$ & $1.21 \pm 0.22$ & $2.96 \pm 0.27$ & $0.50 \pm 0.11$ \\
\hline I & 195202.983 & 261621.05 & $-8.31 \pm 0.04$ & $-8.33 \pm 0.09$ & $3.85 \pm 0.10$ & $2.72 \pm 0.28$ & $3.15 \pm 0.29$ & $0.73 \pm 0.15$ \\
\hline
\end{tabular}

Table 2. Derived parameters of the CO molecular clumps.

\begin{tabular}{lccccccccc}
\hline \hline Clump & $\begin{array}{c}\text { Area } \\
\left(\operatorname{arcmin}^{2}\right) \\
(2)\end{array}$ & $\begin{array}{c}\text { Int } T_{12} \mathrm{CO} \\
\left(\operatorname{arcmin}^{2} \mathrm{~K}\right) \\
(3)\end{array}$ & $\begin{array}{c}\text { Int } T_{13} \mathrm{CO} \\
\left(\operatorname{arcmin}^{2} \mathrm{~K}\right) \\
(4)\end{array}$ & $\begin{array}{c}T_{\mathrm{ex}}(\max ) \\
(\mathrm{K})\end{array}$ & $\begin{array}{c}T_{\mathrm{ex}}(\text { mean }) \\
(\mathrm{K})\end{array}$ & $\begin{array}{c}N_{\mathrm{H}_{2}}(\mathrm{max}) \\
\left(10^{21} \mathrm{~cm}^{-2}\right)\end{array}$ & $\begin{array}{c}N_{\mathrm{H}_{2}}(\operatorname{mean}) \\
\left(10^{21} \mathrm{~cm}^{-2}\right) \\
(8)\end{array}$ & $\begin{array}{c}M_{\mathrm{H}_{2}} \\
\left(M_{\odot}\right) \\
(9)\end{array}$ & $\begin{array}{c}n_{\mathrm{H}_{2}} \\
\left(\mathrm{~cm}^{-3}\right) \\
(10)\end{array}$ \\
\hline $\mathrm{AD}$ & 36.59 & 554.34 & 102.31 & 29.15 & 11.54 & 15.39 & 3.36 & 1438 & 72 \\
$\mathrm{BC}$ & 32.23 & 491.05 & 122.32 & 23.17 & 12.42 & 21.10 & 4.71 & 1775 & 107 \\
$\mathrm{~A}$ & 13.72 & 305.22 & 50.05 & 29.15 & 14.56 & 15.39 & 5.07 & 814 & 177 \\
$\mathrm{~B}$ & 12.63 & 233.59 & 58.97 & 23.17 & 14.73 & 21.10 & 5.69 & 841 & 207 \\
$\mathrm{C}$ & 6.75 & 118.92 & 30.93 & 21.94 & 17.12 & 17.12 & 8.48 & 669 & 423 \\
$\mathrm{D}$ & 2.18 & 37.48 & 6.66 & 20.90 & 15.12 & 8.90 & 5.00 & 127 & 439 \\
$\mathrm{E}$ & 3.70 & 31.23 & 7.71 & 17.10 & 9.28 & 3.71 & 1.25 & 54 & 84 \\
$\mathrm{~F}$ & 3.05 & 40.64 & 7.53 & 17.79 & 12.90 & 4.88 & 2.12 & 76 & 157 \\
$\mathrm{G}$ & 3.70 & 50.50 & 13.38 & 18.95 & 11.19 & 8.42 & 2.53 & 109 & 170 \\
$\mathrm{H}$ & 1.31 & 10.01 & 2.41 & 11.89 & 8.28 & 1.75 & 0.58 & 9 & 66 \\
$\mathrm{I}$ & 2.18 & 27.49 & 5.15 & 13.08 & 8.03 & 3.35 & 1.06 & 27 & 93 \\
\hline
\end{tabular}




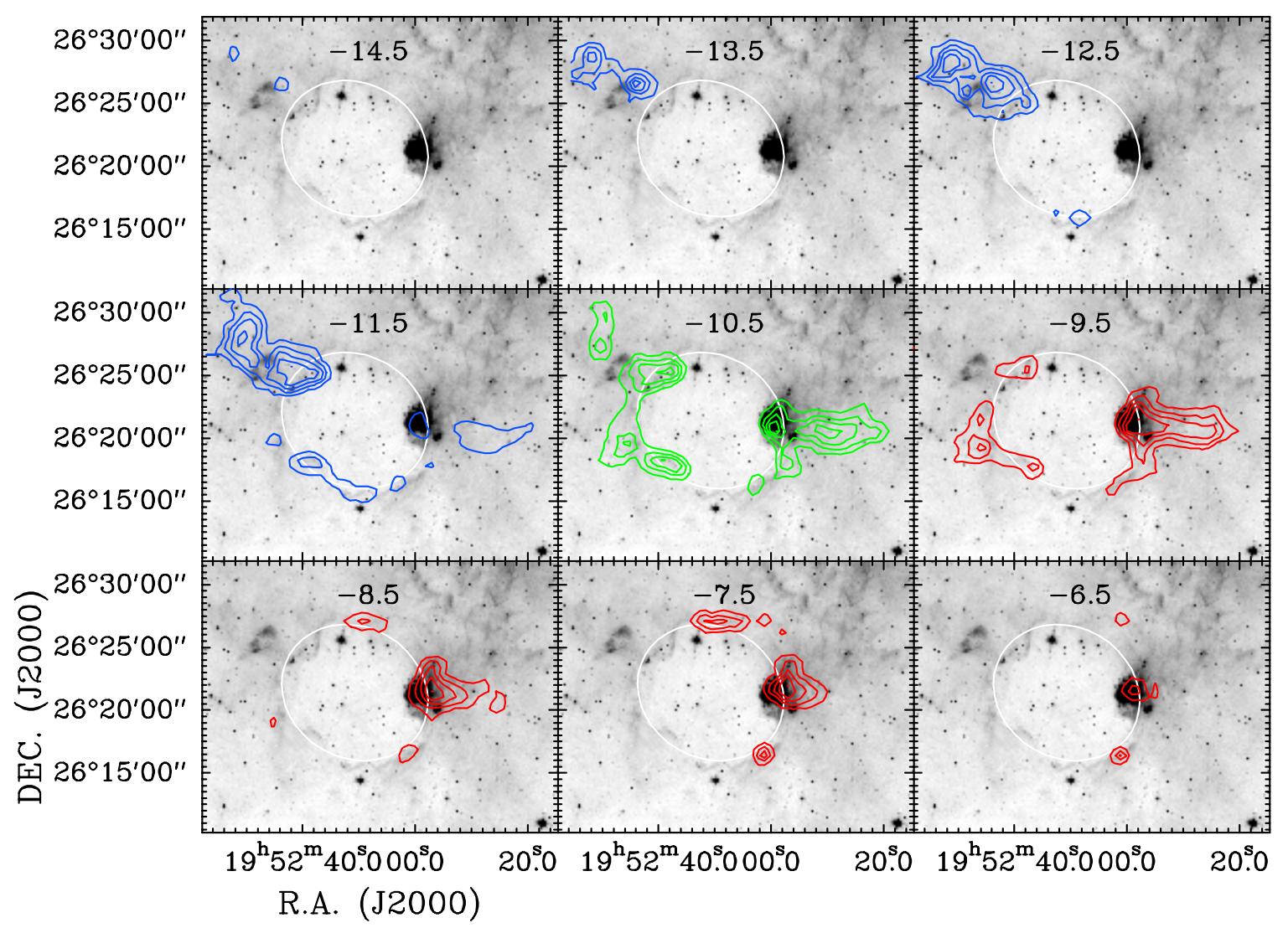

Fig. 4. Integrated intensity contours of the ${ }^{12} \mathrm{CO}$ emission clouds every $1.0 \mathrm{~km} \mathrm{~s}^{-1}$ superimposed on the GLIMPSE $8.0 \mu \mathrm{m}$ grayscale. The lowest contour level for each velocity panel from -14.5 to $-6.5 \mathrm{~km} \mathrm{~s}^{-1}$ is $1.59,1.37,1.80,2.24,1.96,2.19,2.80,1.22$, and $0.95 \mathrm{Kkm} \mathrm{s}^{-1}$, respectively; the level increment of each panel is equal to the corresponding value of the lowest contour level. The blue, green, and red contours indicate the blueshifted, systematic, and redshifted velocities, respectively. The white ellipse indicates the position of bubble N131.

and $\mathrm{BC}$ have extensive structure outwardly. On the north and south of the clump A located at the ringlike shell, there also is an expanding tendency in the western direction. This morphology may be caused by the stellar wind within the bubble, but the possibility needs to be explored further.

Using the channel map (Fig. 4) of ${ }^{12} \mathrm{CO}$ emission to investigate the velocity component of the bubble N131, The green contours in the panel of Fig. 4 show that the systematic velocity of the bubble is about $-10.5 \pm 0.5 \mathrm{~km} \mathrm{~s}^{-1}$, which is consistent with the velocity of the ionized gas $\left(V_{\mathrm{H} 110 \alpha}=-9.3 \pm 2.3 \mathrm{~km} \mathrm{~s}^{-1}\right.$, Watson et al. 2003). The velocity range of the cloud AD is from -9.5 to $-6.5 \mathrm{~km} \mathrm{~s}^{-1}$ indicated with red contours, while the velocity of the cloud $\mathrm{BC}$ is from -14.5 to $-11.5 \mathrm{~km} \mathrm{~s}^{-1}$ indicated with blue contours. We argue that the clouds AD and BC are, respectively, redshifted and blueshifted relative to the ringlike shell of the bubble.

In addition, along the central axis of the clouds $\mathrm{AD}$ and $\mathrm{BC}$ in Fig. 2, we made a position-velocity diagram in Fig. 5. Both the velocity gradient and velocity dispersion gradient are obvious from clumps A (B) to D (C). The velocity of the clump A on the ringlike shell is higher than that of the clump $\mathrm{D}$, which is consistent with the redshifted velocity distribution of the bubble N68 (Zhang \& Wang 2012b). For the cloud BC, the position-velocity diagram shows that clumps $\mathrm{B}$ and $\mathrm{C}$ may be two independent components. However, it is also possible that the molecular clumps $\mathrm{B}$ and $\mathrm{C}$ may be interacting. Figure 6 shows integrated intensity contours of the ${ }^{12} \mathrm{CO}$ emission of the blueshifted (from -14.5 to $-11.5 \mathrm{~km} \mathrm{~s}^{-1}$ ) and redshifted (from -9.5 to $-6.5 \mathrm{~km} \mathrm{~s}^{-1}$ ) clouds. There is almost no overlap region between the blue- and redshifted clouds.

\subsection{Lyman continuum flux}

Figure 6 shows the $1.4 \mathrm{GHz}$ NVSS continuum emission contours. The region of the continuum flux value above $2 \sigma$ noise (or $>0.9 \mathrm{mJy}$ beam $^{-1}$ ) was only integrated to consider as the reliable HII region candidates. From Fig. 6, it can be seen that there is no radio continuum emission within N131, except for a clump projected on the plane of the sky towards the center of the bubble. This fact, along with that the $24 \mu \mathrm{m}$ emission being very weak inside the bubble, would indicate that hot dust and ionized gas have been evacuated by stellar wind (Watson et al. 2009). We also found that IR 2 is located at the peak of HII region G63.049-0.348 (Gregory et al. 1996), suggesting that the IR2 (IRAS 19499+2613) may be the ionizing star of the HII region.

Assuming that the only radio feature in the center of N131 is associated with the bubble, we derive the radio flux density of the HII region in about $0.016 \mathrm{Jy}$. The flux was estimated by integrating each pixel for the signals of more than $2 \sigma$ noise from the NVSS fit image. This value should be taken with caution because the NVSS survey has not added the flux contribution from large-scale structures. The number of stellar Lyman photon, absorbed by the gas in the HII region, follows the relation Eq. (1) in Mezger et al. (1974)

$$
\left[\frac{N_{\mathrm{Lyc}}}{\mathrm{s}^{-1}}\right]=4.761 \times 10^{48} a\left(v, T_{\mathrm{e}}\right)^{-1}\left[\frac{v}{\mathrm{GHz}}\right]^{0.1}\left[\frac{T_{\mathrm{e}}}{\mathrm{K}}\right]^{-0.45}\left[\frac{S_{v}}{\mathrm{Jy}}\right]\left[\frac{D}{\mathrm{kpc}}\right]^{2},
$$

where $a\left(v, T_{\mathrm{e}}\right)$ is a slowly varying function tabulated by Mezger \& Henderson (1967), for effective temperature of ionizing 


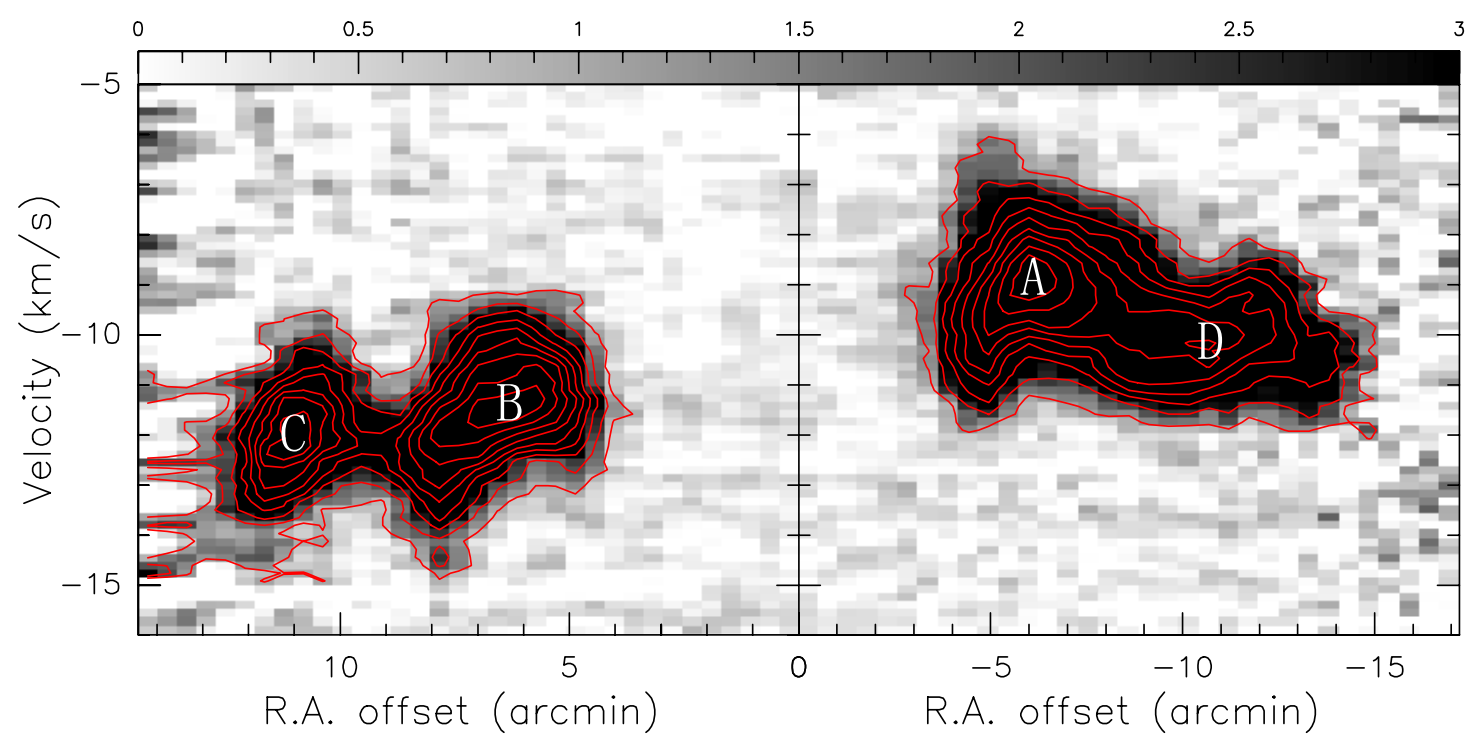

Fig. 5. Position-velocity diagram of the ${ }^{12} \mathrm{CO}$ emission clouds along the white straight lines in Fig. 2. The position corresponding to offset 0 is at $\mathrm{RA}(\mathrm{J} 2000)=19^{\mathrm{h}} 52^{\mathrm{m}} 17 \mathrm{~s}$. , Dec $(\mathrm{J} 2000)=+26^{\circ} 22^{\prime} 05^{\prime \prime} 8$. The position angle of clump BC is positive, and that of clump AD is negative. The contour levels rang from 0.97 to 8.72 by $0.97 \mathrm{~K}$ for the left panel, while from 1.23 to 11.07 by $1.23 \mathrm{~K}$ for the right panel. The color bar of the grayscale for the ${ }^{12} \mathrm{CO}$ emission is above the diagram. The letters A, B, C, and D correspond to the positions of the same letters as in Fig. 2.

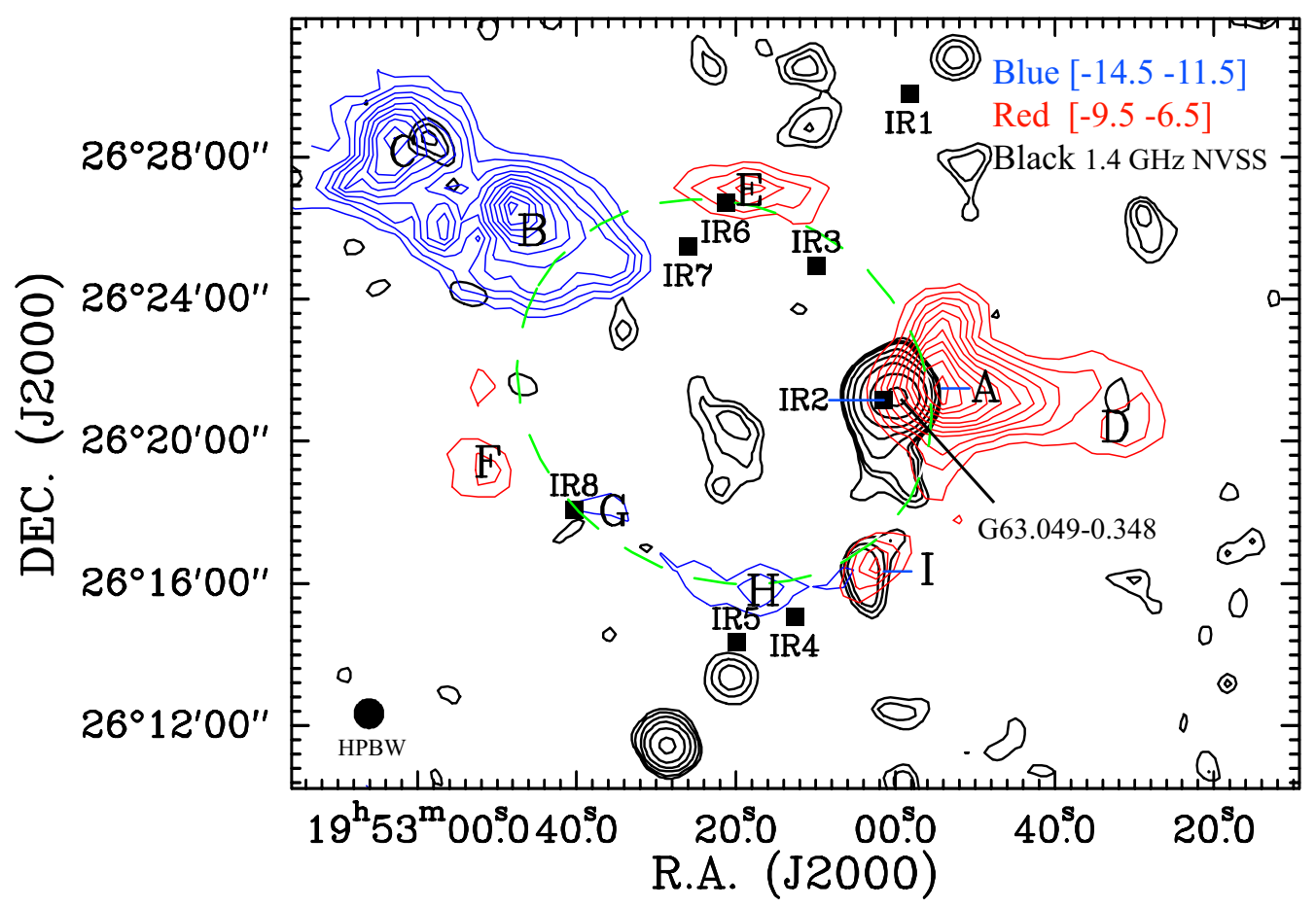

Fig. 6. Integrated intensity contours of the ${ }^{12} \mathrm{CO}$ emission of the blueshifted and redshifted clouds superimposed on the $1.4 \mathrm{GHz}$ NVSS continuum emission contours. The contour levels range from 3.20 to 19.20 by $1.60 \mathrm{~K}$ for the blueshifted cloud, and from 3.86 to 23.17 by $1.93 \mathrm{~K}$ for the redshifted cloud. The integration range is from -14.5 to $-11.5 \mathrm{~km} \mathrm{~s}^{-1}$ for blueshifted cloud, and from -9.5 to $-6.5 \mathrm{~km} \mathrm{~s}^{-1}$ for redshifted cloud. The contour levels of the 1.4 GHz NVSS continuum emission are $0.95,1.26,1.58,3.16,6.32,12.64,25.29$, and $50.58 \mathrm{mJy} \mathrm{beam}^{-1}$. G63.049-0.348 is an HII region. The letters from A to I indicate the positions of nine molecular clumps, and the symbols " $\square$ " indicate the positions of IRAS point sources. The green ellipse indicates the position of bubble N131. The beam size of ${ }^{12} \mathrm{CO}$ emission is given in the filled black circle.

star $T_{\mathrm{e}} \sim 33000 \mathrm{~K}$ and at radio wavelengths, $a\left(v, T_{\mathrm{e}}\right) \sim 1$ Finally, we obtained Lyman continuum ionizing photons flux $\log N_{\text {Lyc }} \sim 46.73$ from nebula. Assuming the ionizing stars belong to the O9.5 star with $\log N_{\text {Lyc }} \sim 47.84$ (Panagia 1973), and the estimated $N_{\text {Lyc }}$ is a factor of 2 lower than the expected ionizing photon flux from these stars (Beaumont \& Williams 2010), it is suggested that there should be about 0.16 ionizing star of 09.5 star to ionize the ISM within the bubble N131.
We also used the Effelsberg radio continuum data from the Galactic plane survey at $2695 \mathrm{MHz}$ (only source component) to derive the Lyman continuum flux(Furst et al. 1990). The beam size of this survey is about $4.3^{\prime}$, rms is $20 \mathrm{mK}$ in brightness temperature, and $T_{\mathrm{B}} / S=2.51 \pm 0.05[\mathrm{~K} / \mathrm{Jy}]$. Within a circle of $2.60^{\prime}\left(0.5 \times r_{\text {in }}\right)$ in radius centered on N131, the average brightness temperature is about $0.3 \mathrm{~K}$, so the intensity is about 0.120 Jy. Using Eq. (1), we obtained the Lyman continuum 
Table 3. IRAS point sources around the bubble N131.

\begin{tabular}{|c|c|c|c|c|c|c|c|c|c|}
\hline Name & $\begin{array}{c}\text { IRAS source } \\
\text { (2) }\end{array}$ & $\begin{array}{c}\mathrm{RA}(\mathrm{J} 2000) \\
\mathrm{h} \quad \mathrm{s} \\
(3)\end{array}$ & $\begin{array}{c}\operatorname{Dec}(\mathrm{J} 2000) \\
\circ, \quad, \\
(4)\end{array}$ & $\begin{array}{l}F_{12} \\
\text { Jy } \\
(5)\end{array}$ & $\begin{array}{l}F_{25} \\
\text { Jy } \\
(6)\end{array}$ & $\begin{array}{l}F_{60} \\
\text { Jy } \\
(7)\end{array}$ & $\begin{array}{l}F_{100} \\
\text { Jy } \\
(8)\end{array}$ & $\begin{array}{l}L_{I R} \\
L_{\odot} \\
(9)\end{array}$ & $\begin{array}{c}T_{d} \\
\mathrm{~K} \\
(10)\end{array}$ \\
\hline IR1 & $19498+2621$ & 195158.18 & 262946.36 & 0.31 & 0.43 & 3.14 & 33.82 & 1855 & 19.47 \\
\hline IR2 & $19499+2613$ & 195201.47 & 262109.56 & 5.54 & 22.02 & 216.90 & 322.80 & 40873 & 32.52 \\
\hline IR3 & $19500+2617$ & 195209.90 & 262456.10 & 0.45 & 0.27 & 3.23 & 322.80 & 13199 & 13.41 \\
\hline IR4 & $19501+2607$ & 195212.62 & 261503.28 & 0.44 & 1.12 & 9.84 & 31.69 & 2627 & 25.78 \\
\hline IR5 & $19502+2606$ & 195219.94 & 261419.75 & 4.14 & 1.70 & 2.99 & 31.51 & 3717 & 19.56 \\
\hline IR6 & $19502+2618$ & 195221.28 & 262641.84 & 0.28 & 0.27 & 2.55 & 53.40 & 2520 & 17.16 \\
\hline IR7 & $19503+2617$ & 195226.01 & 262528.14 & 5.96 & 2.60 & 2.72 & 43.68 & 5149 & 18.01 \\
\hline IR8 & $19505+2610$ & 195240.28 & 261804.06 & 0.30 & 0.22 & 3.38 & 23.33 & 1429 & 21.40 \\
\hline
\end{tabular}
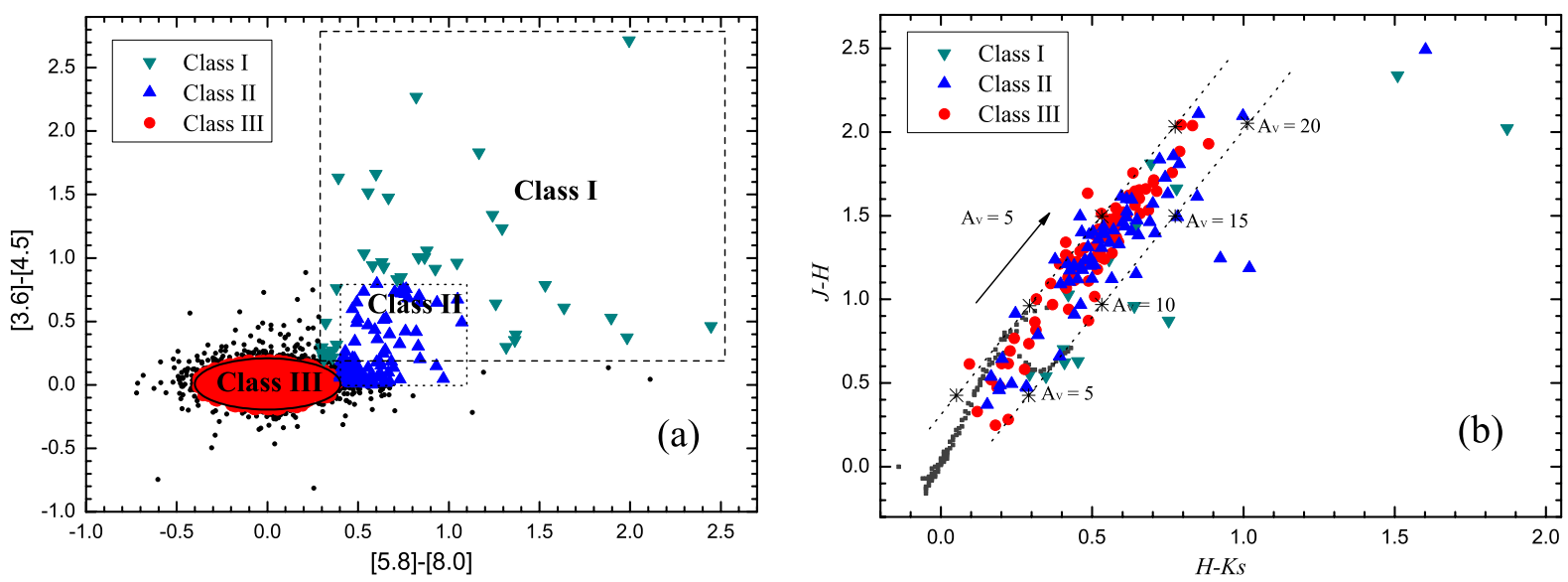

Fig. 7. a) GLIMPSE CC diagram [5.8]-[8.0] versus [3.6]-[4.5] for sources within a circle of about 10' in radius centered on N131. The classification of class I, II, and III indicates different stellar evolutionary stages as defined by Allen et al. (2004). b) 2MASS CC diagram $\left(H-K_{\mathrm{s}}\right)$ versus $(J-H)$. The sources for classes I and II are these, detected simultaneously by $J, H, K_{\mathrm{s}}, 3.6,4.5,5.8$, and $8.0 \mu$ bands; the sources for class III are located within a circle of $2.60^{\prime}\left(0.5 \times r_{\text {in }}\right)$ in a radius centered on N131. The gray squares represent the location of the main sequence and the giant stars (Bessell \& Brett 1988). The parallel dotted lines are reddening vectors. The adopted interstellar reddening law is $A_{J} / A_{V}=0.282$, $A_{H} / A_{V}=0.175$, and $A_{K_{\mathrm{s}}} / A_{V}=0.112$ (Rieke \& Lebofsky 1985), and the intrinsic colors $\left(H-K_{\mathrm{s}}\right)_{0}$ and $(J-H)_{0}$ are obtained from Martins \& Plez (2006).

flux $\log N_{\text {Lyc }} \sim 47.63$. After considering the underestimated factor 2 , we found that there is only about 1.24 ionizing star of 09.5 star to ionize the ISM. Therefore, the Effelsberg radio continuum intensity is also weak like the $1.4 \mathrm{GHz}$ NVSS continuum. Maybe it is not enough to ionize the bubble N131 for the weak continuum intensity.

However, we found 15 reliable ionizing star candidates within the bubble (see Sect. 3.7). It is likely that hot dust and ionized gas have been evacuated by a stellar wind from the clustered ionizing stars, and this will lead to underestimating the Lyman continuum ionizing photons flux from the ionizing stars.

\subsection{IRAS point sources}

Within a circle about $10^{\prime}$ in radius centered on N131, we found eight IRAS point sources, indicated in Figs. 2, 3, and 6 with the names IR1, IR2, ..., and IR8. These IRAS sources, except IR1, are distributed around the several molecular clumps in the line of sight. Especially, IR4, IR6, and IR8 are correlated well with the molecular clumps A, H, E, and G, respectively. We obtained some parameters of the IRAS point sources in Table 3. Columns (1), (2) list the source name; Cols. (3), (4) list the equatorial coordinates; Cols. (5)-(8) list the flux of the 12, 25, 60, and $100 \mu \mathrm{m}$, respectively; Cols. (9), (10) list the derived the infrared luminosity (Casoli et al. 1986) and dust temperature (Henning et al. 1990), which are expressed as

$$
\begin{aligned}
L_{\mathrm{IR}}= & \left(20.653 \times F_{12}+7.538 \times F_{25}+4.578 \times F_{60}\right. \\
& \left.+1.762 \times F_{100}\right) \times D^{2} \times 0.30 \\
T_{\mathrm{d}}= & \frac{96}{(3+\beta) \ln (100 / 60)-\ln \left(F_{60} / F_{100}\right)} .
\end{aligned}
$$

In the equations above, $D$ is the distance from the Sun in kpc, and the emissivity index of dust particles $\beta$ is assumed to be two. Based on the parameters about the infrared luminosity, dust temperature in Table 3, the eight IRAS point sources are probable candidates for young massive stars.

In studying of the occurrence of maser emission from star-forming regions in the very early stages of evolution, Palumbo et al. (1994) found the $22 \mathrm{GHz} \mathrm{H}_{2} \mathrm{O}$ maser emission has a flux of less than $3.1 \mathrm{Jy}$, and van der Walt et al. (1995) did not find any $6.7 \mathrm{GHz}$ methanol maser toward the IR2 (IRAS 19499+2613). Also, in a study surveying the occurrence of the $22 \mathrm{GHz} \mathrm{H}_{2} \mathrm{O}$ maser emission from bright IR sources in star-forming regions, Palla et al. (1991) found the peak flux of $\mathrm{H}_{2} \mathrm{O}$ maser is less than $2.7 \mathrm{Jy}$ toward the IR4 (IRAS 19501+2607) near clump H. 
Table 4. Ionizing star candidates within bubble.

\begin{tabular}{|c|c|c|c|c|c|c|c|c|c|c|c|c|}
\hline GLIMPSE Desig. & $\begin{array}{c}m_{3.6} \\
(\mathrm{mag}) \\
(2) \\
\end{array}$ & $\begin{array}{c}m_{4.5} \\
(\mathrm{mag}) \\
(3) \\
\end{array}$ & $\begin{array}{c}m_{5.8} \\
(\mathrm{mag}) \\
(4) \\
\end{array}$ & $\begin{array}{c}m_{8.0} \\
(\mathrm{mag}) \\
(5) \\
\end{array}$ & $\begin{array}{c}m_{J} \\
(\mathrm{mag}) \\
(6) \\
\end{array}$ & $\begin{array}{c}m_{H} \\
(\mathrm{mag}) \\
(7) \\
\end{array}$ & $\begin{array}{c}m_{K_{\mathrm{s}}} \\
(\mathrm{mag}) \\
(8) \\
\end{array}$ & $\begin{array}{c}A_{V} \\
(\mathrm{mag}) \\
(9) \\
\end{array}$ & $\begin{array}{c}M_{J} \\
(\mathrm{mag}) \\
(10)\end{array}$ & $\begin{array}{c}M_{H} \\
\text { (mag) } \\
(11) \\
\end{array}$ & $\begin{array}{c}M_{K_{\mathrm{s}}} \\
(\mathrm{mag}) \\
(12)\end{array}$ & $\begin{array}{c}\text { O-type star } \\
\text { (13) }\end{array}$ \\
\hline G063.0696-00.3941 & 11.34 & 11.39 & 11.29 & 11.23 & 12.63 & 11.89 & 11.60 & 7.06 & -4.04 & -4.02 & -3.86 & O7V-06.5V \\
\hline G063.0819-00.3947 & 1.01 & 10.94 & 10.88 & 10.89 & 12.65 & 11.77 & 11.29 & 9.26 & -4.64 & -4.52 & & $\mathrm{O} 5.5 \mathrm{~V}-\mathrm{O} 5 \mathrm{~V}$ \\
\hline G063.0918-00.3962 & 11.75 & 11.62 & 11.43 & 11.58 & 13.60 & 12.59 & 12.08 & 10.09 & -3.91 & -3.85 & -3.72 & O7.5V \\
\hline G063.0958-00.4 & 11.75 & 11.82 & 11.65 & 11.67 & 13.70 & 12.51 & 12.08 & 10.29 & -3.88 & -3. & & $\mathrm{O} 7.5 \mathrm{~V}-\mathrm{O} 7 \mathrm{~V}$ \\
\hline G063 & 11.59 & 11.54 & 11.51 & 11.42 & 13.04 & 12.07 & 11.70 & 8.76 & -4.11 & -4.14 & & $07 \mathrm{~V}-06.5 \mathrm{~V}$ \\
\hline G063.1020-00.4375 & 11.55 & 11.60 & 11.48 & 11.43 & 13.51 & 12.32 & 11.87 & 10.45 & -4.12 & -4.18 & & O7V-O6V \\
\hline G063.1050-00.4143 & 12.16 & 12.16 & 11.96 & 12.26 & 14.20 & 12.95 & 12.53 & 10.51 & -3.43 & -3.57 & & $09 \mathrm{~V}-08.5 \mathrm{~V}$ \\
\hline & 10.8 & 10. & 10.7 & 10.71 & 12.61 & & 11. & 10. & -4.89 & & & \\
\hline G063 & 10.73 & 10.65 & 10.60 & 10.46 & 12.31 & 11.37 & 10.94 & 9.05 & -4.92 & -4.89 & -4.74 & O4V-O4V \\
\hline G063.1127-00.4278 & 12.18 & 12.16 & 11.86 & 12.00 & 14.11 & 12.89 & 12.45 & 10.51 & -3.53 & -3.62 & -3.40 & O9V-O8.5V \\
\hline G063.1138-00.4385 & 12.23 & 12.13 & 12.08 & 11.99 & 14.02 & 12.95 & 12.54 & 9.58 & -3.35 & -3.40 & -3.21 & O9.5V-O9V \\
\hline G063.1143-00.3893 & 11.09 & 11.13 & 11.02 & 11.04 & 12.43 & & 11.26 & 7.81 & -4.44 & & & O5.5V-O5V \\
\hline G063.1168-00.4392 & 10.70 & 10.79 & 10.61 & 10.62 & 12.57 & 11.44 & 11.02 & 9.94 & -4.90 & & -4.77 & $\mathrm{O} 4 \mathrm{~V}-03 \mathrm{~V}$ \\
\hline G063.1187-00.4133 & 12.00 & 11.97 & 11.73 & 11.93 & 13.91 & 12.71 & 12.25 & 10.55 & -3.74 & -3.81 & -3.60 & O8V-07.5V \\
\hline G063.1251-00.4258 & 10.71 & 10.73 & 10.54 & 10.57 & 12.56 & 11.43 & 11.00 & 10.03 & -4.94 & -5.00 & -4.80 & $\mathrm{O} 4 \mathrm{~V}-03 \mathrm{~V}$ \\
\hline
\end{tabular}

\section{7. lonizing stars and YSOs}

The GLIMPSE color-color (CC) diagram [5.8]-[8.0] versus [3.6]-[4.5] in Fig. 7a shows the distribution of class I, II, and III stars, which are located within a circle of about $10^{\prime}$ in radius centered on N131. Here we only considered these sources with detection in four Spitzer-IRAC bands (Hora et al. 2008). Class I sources are protostars with circumstellar envelopes; class II sources are disk dominated objects; and class III sources refer to young stars above the main sequence (and contracting towards it), but without accretion characteristics such as $\mathrm{H} \alpha$ emission (Allen et al. 2004; Petriella et al. 2010).

The reliable ionizing stars are mainly from the class III candidates and centrally distributed in a small region within the bubble, so we only considered the sources within a circle of $2.60^{\prime}$ $\left(0.5 \times r_{\text {in }}\right)$ in radius centered on N131. Furthermore to get rid of the background and foreground stars, we used the 2MASS CC diagram $\left(H-K_{\mathrm{s}}\right)$ versus $(J-H)$ in Fig. 7b. Considering the extinction in the Galactic plane as a function of distance to the Sun, Amôres \& Lépine (2005) present an average Galaxy ISM extinction $A_{V}=0.96 \mathrm{mag} \mathrm{kpc}-1$ for one model. The adopted distance of the N131 is $8.6 \mathrm{kpc}$ from the Sun, so we just considered the sources between the extinction range $10.6 \sim 6.6$ mag. Finally, we obtained 15 reliable ionizing star candidates within the bubble N131. In Table 4, we report the 15 ionizing candidates within the bubble: Col. (1) specifies the GLIMPSE designation; Cols. (2)-(8) list the magnitude of four Spitzer-IRAC bands and three 2MASS $J H K_{\mathrm{S}}$ bands, respectively; Col. (9) lists the ISM extinction $A_{\mathrm{V}}$; the absolute $J H K_{\mathrm{s}}$ magnitude and spectral type were derived in Cols. (10)-(13).

YSOs are mainly distributed around the ringlike shell of the bubble, so we selected YSO candidates from class I and class II sources in Fig. 7a. We then excluded the potential sources belonging to the reddening main sequence and giant stars using the 2MASS CC diagram in Fig. 7b. These (class I and II) sources in Fig. 7b only include those with simultaneous detection in the four Spitzer-IRAC bands and three 2MASS $J H K_{\mathrm{s}}$ bands. Finally, we found 29 class I stars and 34 class II stars as YSO candidates, which are indicated around the bubble N131 in Fig. 3. We can see that the clustered YSOs are distributed on the clouds AD and $\mathrm{BC}$ in the line of sight, and several YSOs are located near the molecular clumps $\mathrm{G}$ and $\mathrm{H}$ on the ringlike shell. This distribution provides some evidence for star formation triggered by the bubble. Within the bubble, there are two YSO candidates, which are possibly the background or foreground stars. We also found that there is a good correlation between the YSOs and MIPSGAL $24 \mu \mathrm{m}$ distribution.

\section{Summary}

Based on our CO emission observations of 13.7-m PMO telescope, together with other archival data including GLIMPSE, MIPSGAL, 2MASS, IRAS, and NVSS, we have studied the ISM around the IR dust bubble N131. The main results can be summarized as follows.

1. We found that the ringlike shell of the associated CO clouds is well correlated with the Spitzer 8.0 and $24 \mu \mathrm{m}$ emission, and there are two giant elongated molecular clouds (AD and $\mathrm{BC}$ ) of $\mathrm{CO}$ emission appearing on opposite sides of the ringlike shell of N131. The two elongated clouds may be triggered by the stellar wind from the clustered ionizing stars within the N131, but this possibility needs to be explored further.

2. Contrasting the morphologic distributions between the $\mathrm{CO}$ and $8.0 \mu \mathrm{m}$ emissions, we found that the $\mathrm{CO}$ radii agree rather well with those from the $8 \mu \mathrm{m}$ image. $D_{\mathrm{N} 131}=8.6 \mathrm{kpc}$ was adopted as the distance of the bubble N131 from the Sun.

3. We found there is a cavity within the bubble at $1.4 \mathrm{GHz}$ and $24 \mu \mathrm{m}$, indicating that hot dust and ionized gas have likely been evacuated by stellar wind.

4. Seven IRAS point sources (IR2, IR3, ..., and IR8) are distributed along the ringlike shell of the bubble N131. IR2 (IRAS 19499+2613) is located at the peak of HII region G63.049-0.348. IR2, IR4, IR6, and IR8 are well correlated with the molecular clumps A, H, E, and G, respectively.

5. We found 15 ionizing stars and 63 YSO candidates. The clustered YSOs are distributed along the elongated clouds AD and BC, and several YSOs are located around the clumps G and I. These locations may be the birth places of star formation triggered by the bubble N131. 
Acknowledgements. We wish to thank the anonymous referee and editor Malcolm Walmsley for comments and suggestions that improved the clarity of the paper. We are grateful to the staff at the Qinghai Station of PMO for their assistance during the observations. Thanks go to the Key Laboratory for Radio Astronomy, CAS, for support the operating telescope. This work was supported by the Young Researcher Grant of the National Astronomical Observatories, Chinese Academy of Sciences.

\section{References}

Allen, L. E., Calvet, N., D’Alessio, P., et al. 2004, ApJS, 154, 363

Amôres, E. B., \& Lépine, J. R. D. 2005, AJ, 130, 659

Beaumont, C. N., \& Williams, J. P. 2010, ApJ, 709, 791

Benjamin, R. A., Churchwell, E., Babler, B. L., et al. 2003, PASP, 115, 953

Bessell, M. S., \& Brett, J. M. 1988, PASP, 100, 1134

Carey, S. J., Noriega-Crespo, A., Mizuno, D. R., et al. 2009, PASP, 121, 76

Casoli, F., Combes, F., Dupraz, C., Gerin, M., \& Boulanger, F. 1986, A\&A, 169, 281

Churchwell, E., Povich, M. S., Allen, D., et al. 2006, ApJ, 649, 759

Churchwell, E., Watson, D. F., Povich, M. S., et al. 2007, ApJ, 670, 428

Churchwell, E., Babler, B. L., Meade, M. R., et al. 2009, PASP, 121, 213

Condon, J. J., Cotton, W. D., Greisen, E. W., et al. 1998, AJ, 115, 1693

Dame, T. M., Ungerechts, H., Cohen, R. S., et al. 1987, ApJ, 322, 706

Dame, T. M., Hartmann, D., \& Thaddeus, P. 2001, ApJ, 547, 792

Deharveng, L., Schuller, F., Anderson, L. D., et al. 2010, A\&A, 523, A6

Everett, J. E., \& Churchwell, E. 2010, ApJ, 713, 592

Fazio, G. G., Hora, J. L., Allen, L. E., et al. 2004, ApJS, 154, 10

Furst, E., Reich, W., Reich, P., \& Reif, K. 1990, A\&AS, 85, 805
Garden, R. P., Hayashi, M., Hasegawa, T., Gatley, I., \& Kaifu, N. 1991, ApJ, 374,540

Gregory, P. C., Scott, W. K., Douglas, K., \& Condon, J. J. 1996, ApJS, 103, 427 Henning, T., Pfau, W., \& Altenhoff, W. J. 1990, A\&A, 227, 542

Hora, J. L., Carey, S., Surace, J., et al. 2008, PASP, 120, 1233

Ji, W.-G., Zhou, J.-J., Esimbek, J., et al. 2012, A\&A, 544, A39

Martins, F., \& Plez, B. 2006, A\&A, 457, 637

Mezger, P. G., \& Henderson, A. P. 1967, ApJ, 147, 471

Mezger, P. G., Smith, L. F., \& Churchwell, E. 1974, A\&A, 32, 269

Neugebauer, G., Habing, H. J., van Duinen, R., et al. 1984, ApJ, 278, 1

Palla, F., Brand, J., Comoretto, G., Felli, M., \& Cesaroni, R. 1991, A\&A, 246, 249

Palumbo, G. G. C., Scappini, F., Pareschi, G., et al. 1994, MNRAS, 266, 123

Panagia, N. 1973, AJ, 78, 929

Petriella, A., Paron, S., \& Giacani, E. 2010, A\&A, 513, A44

Rieke, G. H., \& Lebofsky, M. J. 1985, ApJ, 288, 618

Simpson, R. J., Povich, M. S., Kendrew, S., et al. 2012, MNRAS, 424, 2442

Skrutskie, M. F., Cutri, R. M., Stiening, R., et al. 2006, AJ, 131, 1163

van der Walt, D. J., Gaylard, M. J., \& MacLeod, G. C. 1995, A\&AS, 110, 81

Watson, C., Araya, E., Sewilo, M., et al. 2003, ApJ, 587, 714

Watson, C., Povich, M. S., Churchwell, E. B., et al. 2008, ApJ, 681, 1341

Watson, C., Corn, T., Churchwell, E. B., et al. 2009, ApJ, 694, 546

Watson, C., Hanspal, U., \& Mengistu, A. 2010, ApJ, 716, 1478

Werner, M. W., Roellig, T. L., Low, F. J., et al. 2004, ApJS, 154, 1

Winnewisser, G., Churchwell, E., \& Walmsley, C. M. 1979, A\&A, 72, 215

Zavagno, A., Anderson, L. D., Russeil, D., et al. 2010, A\&A, 518, L101

Zhang, C. P., \& Wang, J. J. 2012a, A\&A, 544, A11

Zhang, C.-P., \& Wang, J.-J. 2012b, RAA, 13, 47 